\title{
A Comprehensive Efficient Energy-saving Control Scheme For Transmission Line OnLine Monitoring Device
}

\author{
Ying ZHANG ${ }^{1, a}$, Jie LIU ${ }^{1, b}$, Rui DAI ${ }^{1, c}$, Junjie YANG ${ }^{2, d}$ \\ ${ }^{1}$ China Information \& Telecommunication Company, Sichuan Electric Power Corporation, \\ Chengdu, 610041, China \\ ${ }^{2}$ College of Electronics and Information Engineering, Shanghai University of Electric Power, \\ Shanghai, 200090, China \\ aemail: zhangying@sc.sgcc.com, bemail: dairui_dc@sc.sgcc.com, \\ cemail: yanghongchang@sc.sgcc.com, demail: iamyjj@163.com
}

\begin{abstract}
Keywords: Transmission Line; Online Monitoring; Energy-Saving
\end{abstract}
\begin{abstract}
To improve the overhead transmission line online monitoring device power utilization rate, the online monitoring device is not always collecting data, according to the different operational characteristics of the device units, proposed a comprehensive efficient energy-saving control scheme. Scheme given priority to the adoption with output enable efficient DC-DC converters to provide power for each device, used COMS tubes as the electric control switches for sensors and other high power devices, data acquisition used the strategy of each sensor successively turn on the power, the remote communication went online method combination of serial data trigger and short message trigger, used the controller with low power standby mode, and used the transmission line icing online monitoring device as an example to show the corresponding calculations and tests. The tests show that the test results consistent with the calculated results, using this energy-saving control scheme can effectively reduce the solar panel and battery capacity and volume, improve the power supply reliability, prolong the service life, and reduce the device cost and maintenance cost.
\end{abstract}

\section{Introduction}

With the worldwide power shortage and supply load with electricity load uneven distribution exacerbated, Chinese power system is developing to the UHV, long distance, and large capacity direction, gradually building a strong and smart grid. Improving transmission line reliability and information are the basis for the construction of strong smart transmission grid, therefore, transmission line online monitoring technology has been rapid development and wide application. There are many transmission line online monitoring devices, such as icing[1], galloping[2], insulator contamination[3], insulator leakage current[4].

At present, in China most of online monitoring devices adopt the solar panels and batteries integrated power supply[5]. When adequate light, solar panels priority to the load, the excess electricity to charge the battery, cloudy days or nights battery discharge to the load, due to weather conditions greater impact on the solar panels output power. To meet the long-term reliable operation, have to use large capacity solar panels and batteries[6], while the large capacity of solar panels and batteries quality heavy, high cost, large volume, and inconvenient mounting. To reduce the cost, large capacity batteries generally used lead-acid batteries, as lead-acid battery charged and discharged 300-400 times[7], needed frequent maintenance and replacement, low supply reliability and high cost made lead-acid battery not conducive used and promoted.

In a certain time and weather conditions, power supply obtain limited. How to maximize use the power supply to improve power utilization and reduce the online monitoring device overall power has become one of the key issues, in order to meet the long-term stable operation and low cost requirements, the urgent need is an efficient energy-saving control scheme. Based on the device operational characteristics, proposed an efficient energy-saving control scheme which could greatly 
reduce the solar panel and battery capacity, improve device reliability, in addition, reduce the cost of the device, and has wide application prospects.

\section{Device Structure and Energy Conservation Principle}

Overhead transmission line online monitoring devices generally consist of power subsystem, sensor data acquisition subsystem, communication subsystem, controller subsystem. According to the online monitoring device function determine the sensor types, the controller through short distance communication to control multi sensors to monitor data acquisition, and comprehensively process the data, the result will be sent to remote online monitoring center, power subsystem provides the energy for each part circuit of the online monitoring device.

As the online monitoring device is not real-time acquisition the data, with transmission line icing online monitoring device as an example, device requires acquisition period should be more than 10 minutes and less than 40 minutes, but each period the acquisition operation only need about 1 minute, when collect the data, the device need large power consumption and the rest of the time need small power consumption, according to the different operational characteristics of the device units proposed appropriate energy efficient control scheme to reduce the average power consumption.

\section{Power Subsystem Energy-Saving Control}

Sensors, controllers and other devices required supply voltage determine the power subsystem energy-saving control scheme, device power source can choose solar panels and batteries comprehensive supply mode or inductive energy acquisition and batteries comprehensive supply mode, using high efficient DC-DC converter make device power source convert to sensors, controllers and other components, according to the device operational characteristics, preferences with high conversion efficiency and output enable chip, during acquisition operation not all devices need to run at the same time, therefore, by controlling the power output enable terminal can control part of the circuit electrified, achieving energy-saving purposes.

With icing monitoring device as an example, tension sensor, tilt sensor, temperature and humidity, wind speed and direction integrated ultrasonic sensor use $+12 \mathrm{~V}$ power supply, GPRS unit uses $+5 \mathrm{~V}$, the controller uses $+3.3 \mathrm{~V}$, GPRS unit and the controller unit needs always online, GPRS unit start-up moment required current up to $2 \mathrm{~A}$, but the controller unit power consumption is low with the maximum current less than 100mA. Therefore, select TI's high efficiency converter TPS62133 and TPS62125 respectively for GPRS unit and controller unit. The maximum output current of TPS62133 is up to 3A, and its power conversion efficiency exceeds $90 \%$ with short circuit protection and overheating protection function, the maximum output current of TPS62125 is up to $300 \mathrm{~mA}$, and its power conversion efficiency also exceeds $90 \%$, meeting the power requirements.

\section{Sensor subsystem energy-saving control}

The sensors collect data when power is stable, while data acquisition time accounted for only a small part of the period, so we can control sensor operation by control sensor power. The sensors run only when the data need collected, the rest of the time turn off the power to reduce power consumption, therefore, data acquisition strategy is each sensor successively turn on the power.

With icing monitoring device as an example, since the device power output is $+12 \mathrm{~V}$, and sensors need $+12 \mathrm{~V}$ power supply, considering the voltage drop, conversion efficiency and cost, using TI's CSD25310Q2 P-channel COMS tube as the tension sensor power supply control switch to ensure the sensor normal power supply and improve the conversion efficiency, only when the online monitoring device needs to collect information to provide power for the tension sensor, its circuit schematic shown in Figure 1. 


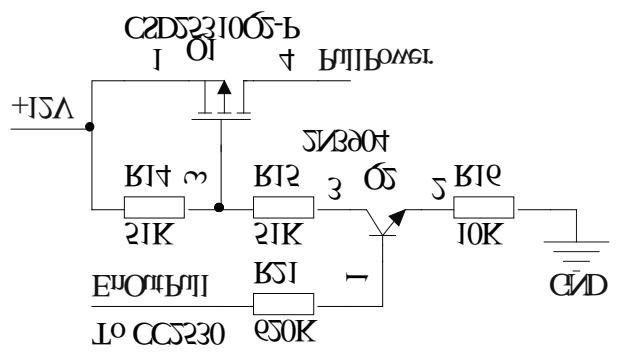

Fig.1. Tension sensor power supply control circuit

As is shown in Figure 4, when EnOutPull is high, 2N3904 transistor is turned on, CSD25310Q2 VGS is $-5 \mathrm{~V}$ and conduction with conduction resistance less then $20 \mathrm{~m} \Omega$, as the conduction current is about $50 \mathrm{~mA}$, so the voltage drop is about $1 \mathrm{mV}$ and sensor supply voltage approximately equal to the power unit output voltage. When EnOutPull is low, 2N3904 and CSD25310Q2 are off, the sensor supply voltage is $0 \mathrm{~V}$.

Considering 2N3904 transistor drive circuit losses and COMS tube conduction losses, the power efficiency $\eta_{1}$ function is as follows:

$$
\eta_{1}=\frac{P_{\text {out }}}{P_{B}+P_{C}+P_{Q}+P_{\text {out }}} \approx \frac{P_{\text {out }}}{P_{C}+P_{\text {out }}}
$$

In the type: $P_{\text {out }}$ is the output power of the sensor power supply control circuit, and the sensor consumption determined the value, $P_{B}$ is 2 N3904 transistor base drive circuit losses, $P_{C}$ is 2 N3904 transistor collector and emitter circuit losses, $P_{Q}$ is COMS tube conduction resistance losses, $P_{B}$ and $P_{Q}$ are relatively small and negligible.

As the practical test, when the input is $11.98 \mathrm{~V}$, if EnOutPull is high, the output voltage is $11.98 \mathrm{~V}$, output power $P_{\text {out }}$ is $605 \mathrm{~mW}, 2 \mathrm{~N} 3904$ transistor collector current is about $0.1 \mathrm{~mA}, P_{C}$ is about $1.2 \mathrm{~mW}$, sensor power supply efficiency is about 99.8\%, if EnOutPull is low, 2N3904 transistor collector current is approximately 0mA, CSD25310Q2 off, the sensor supply voltage is only $0.42 \mathrm{~V}$, so the practical test values are consistent with the theoretical values.

\section{Communication Subsystem Energy-Saving Control}

Remote communication mostly use GPRS wireless communication, online monitoring device and monitoring center are not real-time communication, the GPRS unit consumption with sending and receiving data is up to $2 \mathrm{~W}$, standby consumption without data transceiver is about $0.2 \mathrm{~W}[8]$, try to make GPRS unit running in standby mode to reduce the power consumption.

According to monitoring center commands, online monitoring device needs to periodically sent data to monitoring center, and online monitoring device should immediately response to the set or query commands which come from monitoring center, therefore, use serial data trigger GPRS online when online monitoring device sends data to monitoring center, and use short message trigger GPRS online when monitoring center sends data to online monitoring device, meeting the remote communication requirements and making the GPRS unit maximum in standby mode.

\section{Controller Subsystem Energy-Saving Control}

The online monitoring device is not real-time monitoring, controller use the standby mode to reduce device consumption when it is not monitoring.

With icing monitoring device as an example, controller use ARM or MSP430 processor with standby mode. When set to timing acquisition, the timer interrupt can automatically wake up controller to control data collection, and comprehensively process the data, by serial data triggering GPRS online and sent the results to remote monitoring center, then enter standby mode. In addition, the serial data splitter can wake up the processor when GPRS unit receives a command sent by the remote monitoring center, so that the processor can promptly receive and process set or query commands. 


\section{Energy-Saving Control Test Results}

With icing monitoring device as an example tests the above energy-saving control scheme, by comparing the average power consumption and capacity of solar panels and batteries to test the effect of energy-saving control solutions.

Set the icing online monitoring device minimum acquisition period is 10 minutes, the actual run time per period is 45s. Among them, the tension sensor acquisition time is $26 \mathrm{~s}$ and power consumption is $0.6 \mathrm{~W}$, tilt sensor acquisition time is $8 \mathrm{~s}$ and power consumption is $0.6 \mathrm{~W}$, integrated ultrasonic sensor acquisition time is $4 \mathrm{~s}$ and power consumption is $0.88 \mathrm{~W}$, the controller running time is $45 \mathrm{~s}$ and power consumption is $0.11 \mathrm{~W}$, GPRS communication run time is $12 \mathrm{~s}$ and operating power consumption is $2.15 \mathrm{~W}$ while standby power consumption is $0.18 \mathrm{~W}$, the average power consumption of other circuit is $0.23 \mathrm{~W}$. Without any energy-saving control, calculated with $75 \%$ integrated power supply efficiency, the device average power consumption is $6.09 \mathrm{~W}$, while considering the above efficient energy-saving control scheme, the device average power consumption is $0.548 \mathrm{~W}$, the average power consumption without using energy-saving scheme is 11 times of that with using the above energy-saving scheme, so energy-saving effect of the above energy-saving control scheme is obvious.

Device average power consumption reducing will accordingly reduce solar panel and battery capacity. Device required a minimum capacity of solar panels function $P_{S}$ is as follows:

$$
P_{S}=\frac{P_{L} \times 24}{T_{h} \times \eta_{h}}
$$

In the type: $P_{L}$ is the device average power consumption, $T_{h}$ is the solar panels effective sunshine hours (depending on the region, optional 4-7h), $\eta_{h}$ is the solar panels efficiency (generally $60 \%$ ). The required minimum capacity of battery function $C_{B}$ is as follows:

$$
C_{B}=\frac{P_{L} \times 24 \times N}{U_{B} \times \eta_{B}}
$$

In the type: $U_{B}$ is the battery nominal voltage, $\eta_{B}$ is the battery utilization efficiency (generally $80 \%$ ), $N$ is the longest consecutive days of rain or ice storm (30 days, based on the transmission line equivalent ice thickness online monitoring device technical specifications). Solar panel and battery capacity is proportional to the online monitoring device average power consumption, so the scheme can greatly reduce the solar panel and battery capacity.

Without use of efficient energy-saving control scheme, by Equation 3 the solar panel minimum capacity $P_{S}$ is as follows:

$$
P_{S}=\frac{6.09 \times 24}{4 \times 60 \%}=60.9 \mathrm{~W}
$$

By Equation 3 the battery minimum capacity $C_{B}$ is as follows:

$$
C_{B}=\frac{6.09 \times 24 \times 30}{12 \times 80 \%}=457 \mathrm{Ah}
$$

Therefore, to meet the device power requirements have to use $12 \mathrm{~V} 70 \mathrm{~W}$ solar panels and select 4 sections $12 \mathrm{~V}$ 100Ah lead-acid batteries in parallel. If using the energy-saving control scheme, by Equation 2 the solar panel minimum capacity $P_{S}{ }_{S}$ is as follows:

$$
P_{S}^{\prime}=\frac{0.548 \times 24}{4 \times 60 \%}=5.48 \mathrm{~W}
$$

By Equation 3 the battery minimum capacity $C_{B}{ }_{B}$ is as follows:

$$
C_{B}^{\prime}=\frac{0.548 \times 24 \times 30}{12 \times 80 \%}=41 A \mathrm{~h}
$$

Only use $12 \mathrm{~V} 10 \mathrm{~W}$ solar panels and select 4 sections $12 \mathrm{~V}$ 12Ah iron lithium batteries in parallel. When using iron lithium batteries instead of lead-acid batteries, the battery volume is about $1 / 50$ times of the original volume[7] and the cost is about 2/7 times of the original cost. Therefore, using energy-saving control scheme can improve system reliability and reduce equipment costs. 


\section{Conclusion}

The effect of the energy-saving control scheme proposed in this paper is obvious, after using the energy-saving control scheme, the average power consumption, solar panel capacity and battery capacity are only 1/11 times of without using energy-saving control scheme. Using iron lithium batteries instead of lead-acid batteries, improved the power supply reliability and prolonged the service life, the batteries volume is about 1/50 times of the original volume, convenient device installation in the air and greatly shorten the installation period, the batteries cost is about 2/7 times of the original cost, reduce the device cost and maintenance cost. Therefore, the energy-saving control scheme has broad application prospects in the overhead transmission line online monitoring device.

\section{Acknowledgement}

In this paper, the research was sponsored by National Natural Science Foundation of China (61202369), and Shanghai Technology Innovation Project (13160500900).

\section{References}

[1] Xinbo Huang, Xu Wei. A new online monitoring technology of transmission line conductor icing. Proceedings of 2012 IEEE International Conference on Condition Monitoring and Diagnosis(CMD), 2012, 581-585.

[2] Yusheng Quan, Enze Zhou, Guang Chen. New methodology of online monitoring for transmission line galloping. Energy and Power Technology, 2013,805:867-870.

[3] Yusheng Quan, Ning Chen, Shaigen Han. New methodology of monitoring for the contamination degree of insulators Sustainable Energy, 2014,521:339-342.

[4] Chenguo Yao, Jian Wang, Chengxiang Li. The syntactical pattern recognition for the leakage current of transmissionline insulators. IEEE Transactions on Power Delivery, 2011,26(4):2244-2250.

[5] Rong WU, Jun YANG, Xiaodong LI, Liangzhi WANG. Power solutions of high-voltage online monitoring equipment[J]. Power Supply Technology. 2012,36(5):709-710,737.

[6] Zan WANG, Fei ZONG, Wei WANG, et al. Development of power supply in high voltage side of transmission lines[J]. Advanced of Power System \& Hydroelectric Engineering, 2010, 26(6): 23-27.

[7] Lan XIONG, Youzhong HE, Zhaohui XI, Zikang YANG, Wei HE. Power supply for monitoring equipment based on wind and solar generation system[J]. Power Supply Technology. 2011, 35(7):788-790.

[8] Lan XIONG, Youzhong HE, Daojun SONG, Yong LIU, Wei HE, Zhanlong ZHANG. Design on Power Supply for the Transmission Line Online Monitoring Equipment[J]. High Voltage Engineering. 2010, 9:2253-2257. 